\title{
Tendances d'évolution du peuplement de poissons de la Meuse à Chooz de 1991 à 2008
}

\section{Temporal evolution of fish communities in Chooz in the Meuse River from 1991 to 2008}

\author{
Jeremy Alonso $^{(1)}$, Sébastien Mougenez ${ }^{(2)}$, Cécile Delattre ${ }^{(1)}$ \\ (1) EDF R\&D, Laboratoire National Hydraulique \& Environnement, 6 quai Watier, BP 49, 78401 Chatou Cedex, \\ France \\ cecile.delattre@edf.fr \\ (2) ONEMA, Délégation Interrégionale Nord-Est, 23 rue des Garennes, 57155 Marly, France
}

\begin{abstract}
Résumé - La centrale nucléaire de Chooz sur la Meuse (Ardennes) dispose d'un suivi hydroécologique à l'amont et à l'aval du site depuis une vingtaine d'années. L'objectif de cette étude est d'analyser l'évolution du peuplement de poissons (1991-2008) de la Meuse en liaison avec les conditions hydroclimatiques et la qualité de l'eau. L'un des premiers constats est que la richesse spécifique de la communauté piscicole augmente significativement au cours de cette période aussi bien à l'amont qu'à l'aval de la centrale. Une analyse factorielle des correspondances appliquée à ce suivi permet de mettre en évidence un changement de structure du peuplement piscicole de la Meuse à Chooz au cours de la période 1991-2008. L'analyse de l'écologie des espèces caractérisant la communauté indique une évolution vers des espèces plus exigeantes vis-à-vis de leur milieu et plutôt caractéristiques des zones supérieures des cours d'eau telles que le vairon, le chabot et la loche franche. L'implication de la bouvière dans l'évolution de la structure du peuplement piscicole est également très forte. Les modifications sur le long-terme de la composition du peuplement de poissons semblent plutôt expliquées par l'amélioration de la qualité physico-chimique de la Meuse que par les conditions hydroclimatiques.
\end{abstract}

Mots-clés - poissons, Meuse, évolution hydroclimatique, qualité d'eau, suivi long terme

Abstract - A hydrobiology monitoring has been carried out around the Chooz nuclear power plant located on the Meuse River (Ardennes) for about twenty years. The aim of this study is to analyze the temporal evolution of the Meuse River fish community (1991-2008) in relation to hydroclimatic and water quality parameters. One of the results is that the specific richness has significantly increased during this period upstream and downstream of the site. A Correspondence Analysis performed on these data highlighted a change in the fish community structure in the two stations. According to the ecology of the species, there is an evolution towards fish more sensitive to the environment and characteristic of upstream rivers like eurasian minnow, bulhead and stone loach. Moreover, bitterling is also responsible for 
this change. The changes observed in the long-term biological series seem to be explained by the improvement of the water quality rather than the hydroclimatic variability.

Key words - fish, Meuse River, hydroclimatic tendance, water quality, long-term series

\section{INTRODUCTION}

La mise en place de la Directive Cadre sur l'Eau en 2000 et la canicule de 2003 ont notamment remis sur le devant de la scène les questionnements liés aux rejets thermiques dans les cours d'eau inhérents au fonctionnement des centrales thermiques à flamme et nucléaires. Les scientifiques, confrontés à cette problématique dès les années 1970, ont mis en place des suivis au droit de chaque site nucléaire, qui sont maintenant très utiles pour comprendre les évolutions biologiques et tenter de les relier à la fois aux facteurs généraux caractérisant les fleuves récepteurs et aux impacts plus localisés des installations industrielles.

L'analyse de telles séries long terme, rares au niveau international, est riche d'enseignements sur l'effet des forçages climatiques (Delattre \& Souchon, 2008 ; Seegert et al., 2008), et nécessaire à la compréhension des effets du réchauffement généralisé réévalué à environ $+0,74^{\circ} \mathrm{C}$ depuis la fin du $\mathrm{XIX}^{\mathrm{e}}$ siècle (IPCC, 2001, 2007, 2008). D'autant plus que si des travaux ont déjà été engagés concernant l'impact du réchauffement climatique sur divers écosystèmes (Hughes, 2000 ; Walther et al., 2002 ; Parmesan \& Yohe, 2003), peu concernent les milieux aquatiques continentaux.

De longues chroniques de peuplements aquatiques (macroinvertébrés, poissons) de grands fleuves et estuaires français ont été analysées récemment par Daufresne et al. (2004), Delpech (2007), Daufresne \& Boët (2007), Dessaix \& Fruget (2008), Tales (2008), Khalanski et al. (2008).

L'objectif de cette étude est de compléter les analyses déjà effectuées sur le Rhône, la Seine et la Loire en traitant les données long-terme d'inventaires piscicoles réalisés dans la Meuse de 1991 à 2008 au droit de la centrale nucléaire de Chooz en lien avec les paramètres environnementaux du milieu.

\section{MATÉRIEL ET MÉTHODES}

\subsection{Présentation du site}

La centrale nucléaire est située sur la commune de Chooz près de la frontière belge, dans le département des Ardennes, entre Charleville-Mézières (France) et Dinant (Belgique). Le site nucléaire de Chooz est constitué de :

- Chooz A en rive droite de la Meuse qui comporte une tranche nucléaire de 305 MW mise en service en 1967 et définitivement arrêtée en 1991 ;

- Chooz B en rive gauche de la Meuse constituée de deux tranches nucléaires de $1450 \mathrm{MW}$ chacune, mises en service respectivement en 1996 et 1997 et équipées d'aéroréfrigérant. 


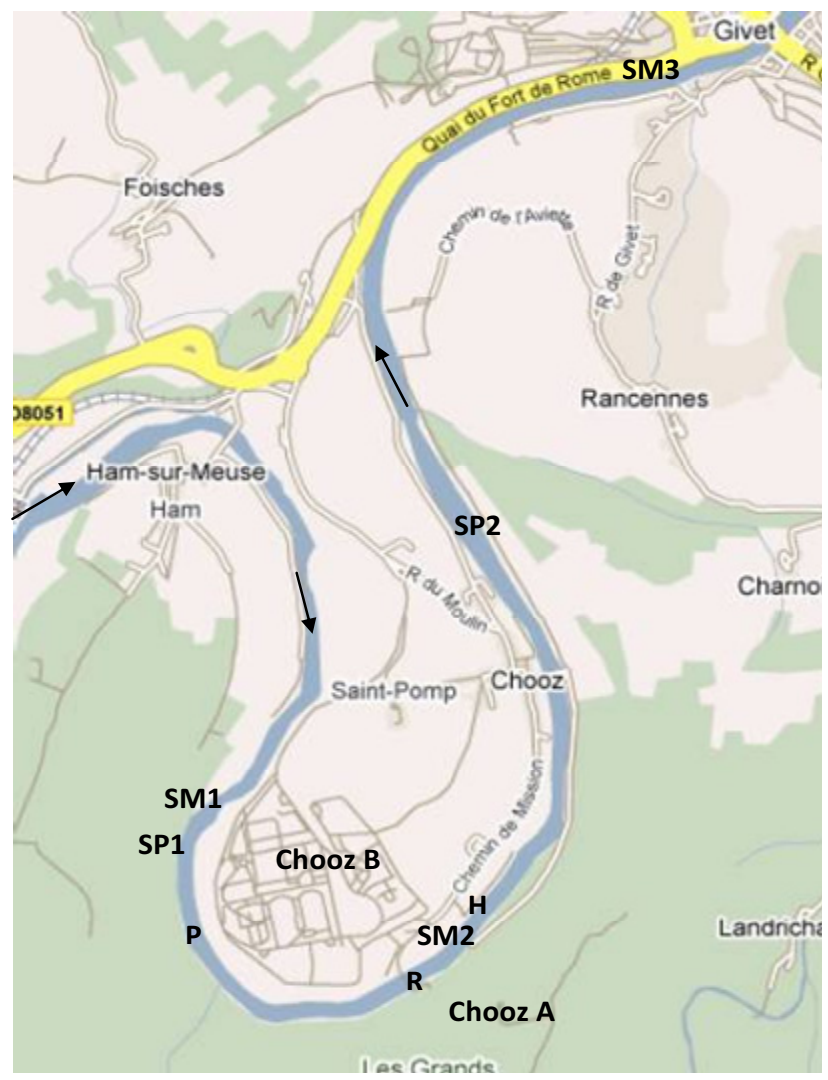

Fig. 1. Localisation de la centrale de Chooz et des stations d'étude.

$\mathrm{P}$ : prise d'eau, $\mathrm{R}$ : rejet, $\mathrm{H}$ : station hydrologique, $\mathrm{SM}$ : station multiparamètres $1:$ amont, 2 : aval proche, $3:$ aval après mélange, SP : station de pêche $1:$ amont, $2:$ aval, $\rightarrow:$ sens d'écoulement de la Meuse. Échelle : $1,7 \mathrm{~cm}=1 \mathrm{~km}$ (source : http://maps.google.fr/).

Fig. 1. Location of Chooz power plant and the study sites.

$\mathrm{P}$ : water intake, $\mathrm{R}$ : discharge point, $\mathrm{H}$ : hydrological station, $\mathrm{SM}$ : multi-parameters station 1 upstream, 2 downstream close to the discharge point, 3 : downstream after mixing, SP : sampling station 1 upstream, 2 downstream, $\rightarrow$ flow direction.

La Meuse prend sa source dans un bassin sédimentaire (plateau de Langres à environ $350 \mathrm{~m}$ d'altitude) et draine jusqu'à la frontière franco-belge un important bassin versant dont une partie est constituée par des terrains primaires (grès et schistes) mais où les calcaires dominent près de $50 \%$ de la superficie. Après un cheminement en
France et en Belgique, et après un parcours de $950 \mathrm{~km}$, la Meuse se jette dans la mer du Nord aux Pays-Bas, son embouchure étant confondue avec les divers bras du Rhin.

La centrale de Chooz se trouve dans une boucle non navigable de la Meuse (Fig. 1). Un canal de dérivation, comportant deux écluses, court-circuite la 
boucle depuis le barrage de Ham-surMeuse jusqu'au lieu-dit « les trois fontaines". Le barrage de Ham-surMeuse a pour fonction de réguler le débit de la Meuse. Il permet le trafic fluvial en accédant au port de Givet tout en maintenant un débit suffisant dans la boucle et nécessaire à la centrale. La prise d'eau en Meuse pour le refroidissement de la centrale de Chooz est située en rive gauche de la Meuse et en amont du seuil de l'ancienne centrale Chooz $\mathrm{A}$ à $1,3 \mathrm{~km}$ environ.

Les dispositions réglementaires à respecter par l'exploitant du site de Chooz B concernant l'environnement aquatique sont définies dans l'arrêté d'autorisation de prise d'eau et de rejets d'effluents de novembre 2009 (MEEDDM).

\subsection{Données environnementales}

Les stations multiparamètres amont (SM1), aval immédiat (SM2) et aval lointain (SM3) gérées par la centrale nucléaire de Chooz mesurent en continu la température de l'eau (Fig. 1). Les températures aquatiques utilisées ici sont disponibles en moyenne journalière de 1997 à 2007 . Certaines années (en particulier 2003 et 2005), les chroniques de température ne sont pas complètes. En outre, la station SM2 (aval proche) fournit des données incohérentes pour plusieurs années car elle a été exondée. Les données de cette station ne sont pas utilisées.

Les mesures journalières de débit de la Meuse sont librement accessibles sur Internet, de 1953 à 2008, grâce à la Banque HYDRO du Ministère de l'Écologie. La station hydrologique Graviat la plus proche du site de Chooz se situe à l'aval de la centrale (Fig. 1). La validité des données de cette station est qualifiée de «bonne" sur la banque HYDRO. Le débit moyen inter-annuel de la Meuse au niveau de la centrale est de $144 \mathrm{~m}^{3} / \mathrm{s}$.

Des analyses mensuelles de matières en suspensions (MES), $\mathrm{pH}$, demande biologique en oxygène $\left(\mathrm{DBO}_{5}\right)$, demande chimique en oxygène (DCO), d'azote et de phosphore total sont disponibles de 1995 à 2007 au niveau des stations amont SM1 et aval SM2, SM3 excepté pour l'azote total dont les données sont disponibles depuis 1997.

\subsection{Données piscicoles}

Les inventaires piscicoles sont réalisés dans le cadre de la surveillance de l'environnement de la centrale nucléaire de Chooz. Les pêches sont menées lors de deux campagnes annuelles en période de basses eaux, l'une au printemps et l'autre à l'automne. Elles sont réalisées à l'électricité à pied et/ou en bateau (suivant le faciès) par l'Office national de l'eau et des milieux aquatiques (ONEMA délégation Alsace, Lorraine, ChampagneArdennes) depuis 1991, avec l'appui de prestataires au niveau de deux stations : une à l'amont et une à l'aval de la centrale (Fig. 1 et Tab. I).

La station de pêche amont (SP1) est prospectée en continu sur certaines portions de berge jusqu'en 1997 puis par ambiances jusqu'à 2006 sur une douzaine de points représentant majoritairement les berges. La pêche représente une surface prospectée de 
Tableau I. Présentation des stations de pêche (Pihan, 2003 ; Dubost, 2009).

Table I. Presentation of fish sampling stations (Pihan, 2003; Dubost, 2009).

\begin{tabular}{lcc}
\hline Caractéristiques & Station de pêche SP1 & Station de pêche SP2 \\
\hline Abscisse (Lambert 2 étendu, $\mathrm{m})$ & 775441 & 776612 \\
Ordonnée (Lambert 2 étendu, $\mathrm{m})$ & 2569432 & 2571175 \\
Situation par rapport au CNPE & Amont & Aval \\
Distance au CNPE $(\mathrm{km})$ & $\sim 2$ & $\sim 2,8$ \\
Distance à la source $(\mathrm{km})$ & 476 & 480 \\
Altitude $(\mathrm{m})$ & 104 & 101 \\
Pente $(\%)$ & 0,3 & 0,3 \\
Surface du bassin versant drainé $\left(\mathrm{km}^{2}\right)$ & 10387 & 10400 \\
Fréquentation par les pêcheurs & Modérée & Modérée \\
Catégorie piscicole & 2 & 2
\end{tabular}

Portion plutôt sinueuse, peu ombragée avec quelques abris pour les poissons. Mêmesilefa- Portion rectiligne sans ciès est principalement

Description profond peuplé de phanérogames, il existe nérogames où les des zones plates moins abris pour les poissons profondes et au courant sont rares. plus vif avec peu de végétation.

l'ordre de $900 \mathrm{~m}^{2}$. La station de pêche aval (SP2) est, jusqu'en 2006, prospectée en continu sur les berges sur environ $1500 \mathrm{~m}^{2}$ autour d'une île (au niveau du lieu dit «Les onze verges »).

À partir de 2007, les deux stations sont prospectées par la méthode d'échantillonnage ponctuel d'abondance (EPA). La technique de l'EPA repose sur l'échantillonnage d'un grand nombre de points répartis de manière aléatoire sur la station étudiée. L'objectif est de procéder à un échantillonnage le plus représentatif possible de la portion de cours d'eau étudiée. La richesse spécifique peut alors être comparée aux pêches à l'électricité des années précédentes mais pas les effectifs bruts. C'est pourquoi, et par souci d'homogénéité avec les études précédemment réalisées dans le domaine (Daufresne et al., 2004 ; Cattanéo et al., 2001 ; Grenouillet et al., 2001), il a été choisi de normaliser l'ensemble des effectifs par rapport au temps de pêche de chaque campagne. Ainsi un effectif dans cette étude représentera toujours un effectif normalisé par le temps, soit un nombre d'individus pêchés en 20 min. 


\subsection{Traitement des données et analyses statistiques}

\subsubsection{Traitement et analyse préalable du contexte abiotique}

Les données journalières de débit sont moyennées sur l'ensemble de l'année pour toute la chronique. Une visualisation graphique du débit moyen sera présentée pour chaque année de la chronique ainsi que les minima et maxima journaliers associés et les boîtes à moustaches permettant la représentation des statistiques classiques de l'ensemble des données.

Les températures de l'eau annuelles moyennes sont calculées à partir des données journalières et analysées aux stations multiparamètres SM1 (amont) et SM3 (aval lointain).

Les données de débit et de température de l'eau sont traitées sur l'ensemble de l'année, pour la saison printanière (avril-juin), période de reproduction de la majorité des espèces de cyprinidés présentes et pour la saison estivale (juillet-octobre), période de développement des juvéniles de la majorité des espèces de cyprinidés présentes.

Les paramètres de qualité d'eau (MES, pH, $\mathrm{DBO}_{5}, \mathrm{DCO}$, azote total et phosphore total) sont analysés annuellement au niveau des trois stations multiparamètres.

\subsubsection{Traitement et analyse préalable} de la communauté piscicole

Les pêches effectuées entre fin août et octobre sont traitées dans un premier temps car elles permettent le recensement des cyprinidés juvéniles nés pendant l'été (Daufresne et al., 2004 ; Tales, 2008). Les pêches printanières sont analysées dans un second temps afin d'étudier les éventuelles différences.

Les espèces peu présentes (en fréquence ou en effectif) n'ont pas été considérées. Un premier tri permet de ne garder que les espèces présentes au moins trois fois dans la chronique (Tales, 2008) et dont l'effectif total sur l'ensemble de la série dépasse trois individus. Les espèces dont la fréquence est supérieure à $5 \%$ des pêches sont retenues dans un premier temps (Daufresne et al., 2004). Très peu d'espèces répondant à ce critère, le seuil de $1 \%$ est proposé. Pour ce critère (FR1), il est important de prendre en compte l'ensemble de la chronique mais également de la morceler en deux, pour ne pas passer à côté d'espèces à l'effectif non négligeable mais présentes uniquement en début ou fin de chronique et donc intéressantes pour l'analyse. On doit finalement veiller à ce que l'ensemble des espèces conservées représentent au moins $95 \%$ de l'effectif total (Daufresne et al., 2004) pour s'assurer de considérer les espèces réellement constitutives de la communauté.

Les paramètres classiques décrivant la communauté piscicole sont le nombre d'espèces et l'équitabilité qui représente la régularité de l'abondance relative des espèces présentes (Tab. II).

Afin de constater l'influence éventuelle de l'effort de pêche sur la qualité d'échantillonnage dans le temps, les effectifs et la richesse spécifique sont représentés graphiquement en parallèle de l'effort de pêche. 
Tableau II. Formule de calcul de l'équitabilité.

Table II. Calculation of eveness.

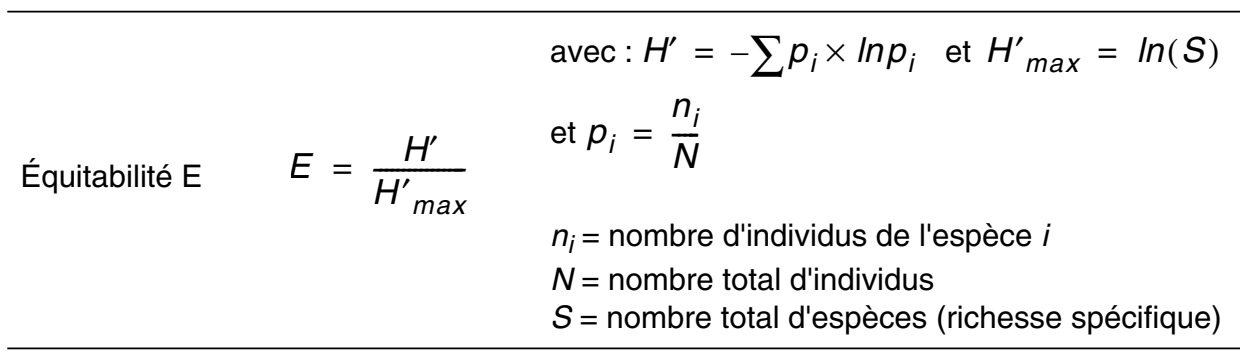

Les méthodes d'analyses en axes principaux (méthodes factorielles) sont réalisées avec le logiciel libre $R$ (http:// www.r-project.org/) à l'aide des packages ade 4 et ade4TkGUI (Thioulouse \& Dray, 2007 ; Chessel et al., 2004). Afin d'homogénéiser leur distribution et de réduire le poids des espèces abondantes, les effectifs utilisés pour les analyses subissent une transformation logarithmique $\ln (x+1)$ (Daufresne et al., 2004).

\subsubsection{Analyse des tendances d'évolution}

À titre indicatif les tendances sont dans un premier temps calculées par une simple régression linéaire afin d'observer le signe et l'intensité de celle-ci.

La tendance d'évolution pour chaque variable (paramètres environnementaux et biologiques) est ensuite évaluée par la statistique $S$ de MannKendall (Daufresne, 2004, 2008; Delpech, 2007). Pour une série d'observations $X=x_{1}, x_{2}, \ldots, x_{n}$, le test classique de Mann-Kendall consiste à calculer la statistique $S$ tel que :

$$
S=\sum_{i<j} \operatorname{sgn}\left(x_{j}-x_{j}\right)
$$

où

$$
\operatorname{sgn}\left(x_{j}-x_{i}\right)=\left\{\begin{array}{c}
1 \text { si } x_{i}<x_{j} \\
0 \text { si } x_{i}=x_{j} \\
-1 \text { si } x_{i}>x_{j}
\end{array}\right.
$$

et $\operatorname{var}(S)=\frac{n(n-1)(2 n+5)}{18}$.

La présence d'une tendance significative est testée en comparant la statistique $Z=\frac{S}{\sqrt{\operatorname{Var}(S)}}$ avec la loi normale. Cependant, lors de l'étude de séries temporelles telles que les nôtres, les observations ne sont pas indépendantes, ce qui peut conduire, à tort, à la conclusion d'une tendance significative. Pour prendre en compte ces autocorrélations temporelles, la variance est corrigée suivant la méthode développée par Hamed \& Rao (1998). II s'agit de soustraire de la série $X$ une estimation non paramétrique de la tendance Sen (1968). Les autocorrélations entre les rangs de cette nouvelle série sont alors calculées et les coefficients d'autocorrélation significativement différents de zéro $\left(\rho_{S}(I)\right.$ au pas de temps i) sont utilisés afin d'apporter une correction à la variance de $S$ :

$$
V^{*}(S)=\operatorname{var}(S) \times \text { Cor }
$$




$$
\begin{aligned}
& \text { où } \\
& \quad \operatorname{Cor}=1+\frac{2}{n(n-1)(n-2)} \\
& \times \sum_{i=1}^{n-1}(n-1)(n-i-1)(n-i-2) \times \rho_{s}(i) .
\end{aligned}
$$

\subsubsection{Analyse des corrélations}

Les corrélations entre paramètres environnementaux et biologiques sont calculées à l'aide du coefficient de corrélation de Pearson (Daufresne, 2008; Delpech, 2007) mais, comme dans l'analyse des tendances, les autocorrélations existantes dans les séries temporelles peuvent entraîner des conclusions erronées concernant l'existence significative d'une corrélation. Un ajustement du nombre de degrés de liberté en fonction des autocorrélations temporelles permet, selon Pyper \& Peterman (1998) de remédier le plus efficacement au problème. Pour des séries de longueur $N$, le nombre de degrés de liberté $\left(N^{*}-2\right)$ est évalué en fonction des $N / 5$ premiers coefficients d'autocorrélation $r_{x x}(\lambda)$ des deux séries :

$\frac{1}{N^{*}} \approx \frac{1}{N}+\frac{2}{N} \times \sum_{j=1}^{N / 5} r_{X X}(j) \times r_{Y Y}(j)$,

avec

$$
r_{x x}(j)=\frac{N}{N-j} \times \frac{\sum_{t=1}^{N-j}\left(x_{t}-\bar{x}\right)\left(x_{t+j}-\bar{x}\right)}{\sum_{t=1}^{N}\left(x_{t}-\bar{x}\right)^{2}} .
$$

Ainsi, la probabilité de signification du coefficient de corrélation de Pearson est calculée grâce à une loi de Student à $N^{*}-2$ degrés de liberté.

\section{RÉSULTATS}

\subsection{Paramètres hydroclimatiques}

\subsubsection{Débit de la Meuse}

Sur l'ensemble de la chronique 1991-2008, les débits moyens annuels sont stables et proches du module inter-annuel de $144 \mathrm{~m}^{3} / \mathrm{s}$ (Figs. 2, 3, Tab. III). Les minima ainsi que le quantile à $25 \%$ sont en augmentation significative tandis que l'écart type à la moyenne diminue significativement.

Au-delà des considérations statistiques, on peut noter que l'enchaînement des années 1995 et 1996 est très contrasté. 1995 étant une année à paramètres élevés tandis que 1996 est une année à paramètres bas ; ceci est notamment très visible concernant les maxima (le début de la chronique, au travers de 1991, 1993 et 1995, montre un grand nombre de jours à fort débit).

De 1998 à 2002 le débit augmente progressivement pour finalement connaître une période basse de 2003 à 2005. Même si les maxima ne présentent pas de tendance significative sur l'ensemble de la chronique, ils sont ponctuellement très élevés en 1993 et 1995 (supérieurs au débit de crue centennal de $1385 \mathrm{~m}^{3} / \mathrm{s}$ ) ainsi qu'en 1991 et 2003 (supérieurs au débit de crue décennal de $1041 \mathrm{~m}^{3} / \mathrm{s}$ ) et très bas en 1996, 2005 et 2006.

Les minima ont une tendance significative à la hausse, avec des valeurs très basses en 1991, 1996, 2005 (inférieures au débit d'étiage de $20 \mathrm{~m}^{3} / \mathrm{s}$ ) et élevées pour les années 1995, 2000 et 2008.

On constate donc que l'année 1995 se caractérise par des paramètres de 


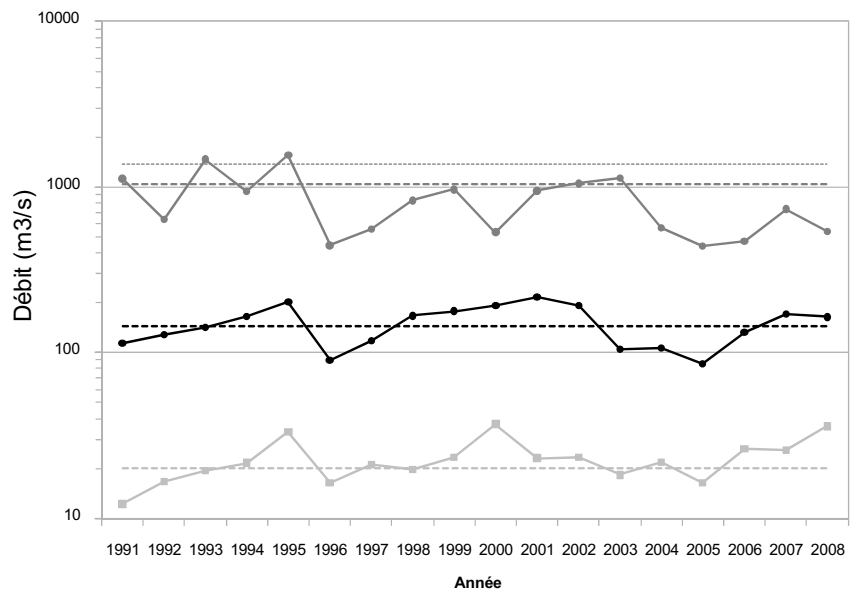

Fig. 2. Évolution des débits journaliers moyennés sur l'ensemble de l'année $\left(\mathrm{m}^{3} / \mathrm{s}\right)$ de la Meuse à Chooz de 1991 à 2008. En gris clair : débit minimal, noir : débit moyen, gris foncé : débit maximal.

Fig. 2. Evolution of daily discharges averaged over the year $\left(\mathrm{m}^{3} / \mathrm{s}\right)$ at Chooz from 1991 to 2008 . Minimum discharge (grey line), mean discharge (black line) and maximum discharge (dark grey line).

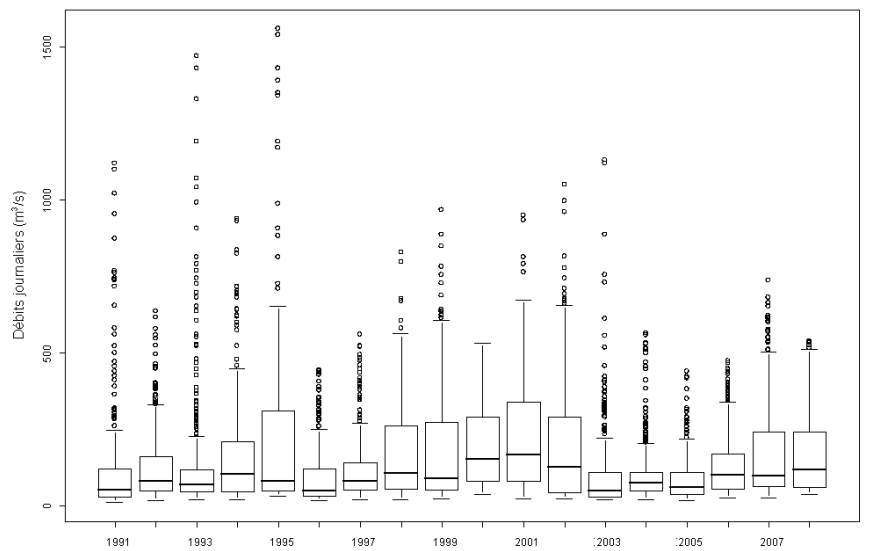

Fig. 3. Boîtes et moustaches du débit journalier $\left(\mathrm{m}^{3} / \mathrm{s}\right)$ de la Meuse à Chooz de 1991 à 2008.

Fig. 3. Boxes and wiskers of daily discharge $\left(\mathrm{m}^{3} / \mathrm{s}\right)$ at Chooz from 1991 to 2008.

débit élevés (extrême et moyenne) alors qu'à l'inverse en 1996 et de 2003 (quelques maxima) à 2005 les paramètres sont très bas (extrême et moyenne).
L'année 2000 apparaît comme une année particulière car la moyenne est plus élevée que le module inter-annuel, les minima sont élevés et les maxima faibles. 
Tableau III. Résultats des tendances d'évolution des débits journaliers de la Meuse à Chooz moyennés sur l'année de 1991 à 2008.

Table III. Results of trend tests of annual discharge at Chooz station from 1991 to 2008.

\begin{tabular}{lccc}
\hline Débit & Tendance & $p$-value & Significativité \\
\hline Maximum & & 0,1396 & \\
Quantile $75 \%$ & & 0,7048 & \\
Quantile $50 \%$ (Médiane) & & 0,1849 & \\
Moyen & $\nearrow$ & 0,6769 & \\
Écart type au débit moyen & $\nearrow$ & 0,0452 & $*$ \\
Quantile $25 \%$ & $\nearrow$ & 0,0177 & $*$ \\
Minimum & & 0,1033 & \\
\hline Nombre de jours de l'année où le débit & & & \\
journalier est inférieur à l'étiage $\left(20 \mathrm{~m}^{3} / \mathrm{s}\right)$ & & 0,2720 & \\
Nombres de jours de l'année où le débit & & & \\
journalier est supérieur à $1000 \mathrm{~m}^{3} / \mathrm{s}$ & &
\end{tabular}

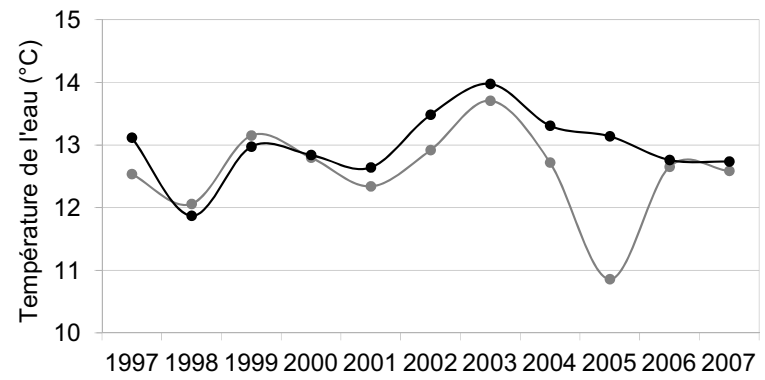

Fig. 4. Évolution des températures moyennes annuelles $\left({ }^{\circ} \mathrm{C}\right)$ de la Meuse à Chooz de 1997 à 2007 à l'amont (SM1 gris clair) et à l'aval éloigné (SM3 noir).

Fig. 4. Annual evolution of water temperature $\left({ }^{\circ} \mathrm{C}\right)$ at Chooz from 1997 to 2007 at upstream (SM1 grey line) and further downstream (SM3 black line) stations.

L'analyse des débits durant la période printanière (avril-juin) et estivale (juillet-octobre) ne montre aucune tendance significative. La période 2003-2005 de bas débits observée à l'échelle annuelle est également visible aux saisons printanières et estivales.

\subsubsection{Température de la Meuse}

On rappelle tout d'abord que les températures de la station aval proche
(SM2) n'ont pas pu être traitées compte tenu d'un problème de validité des données (sonde exondée). La température moyenne annuelle de l'eau à l'amont et l'aval éloigné de la centrale oscille entre 12 et $13^{\circ} \mathrm{C}$ de manière constante tout au long de la chronique, l'année 2003 étant la plus chaude (Figs. 4 et 5).

On note un écart fortement marqué entre l'amont et l'aval en 2005. II s'agit en fait de l'année où il manque le plus de données de température de l'eau en 


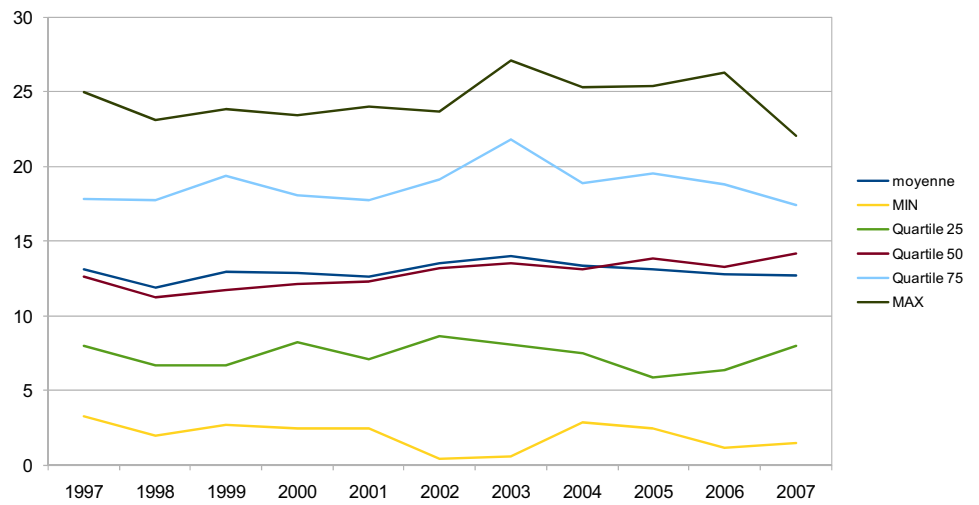

Fig. 5. Évolution des températures de la Meuse $\left(\mathrm{en}^{\circ} \mathrm{C}\right)$ de 1997 à 2007 à la station aval éloigné SM3. Fig. 5. Annual evolution of water temperature $\left({ }^{\circ} \mathrm{C}\right)$ at further downstream station (SM3) from 1997 to 2007.
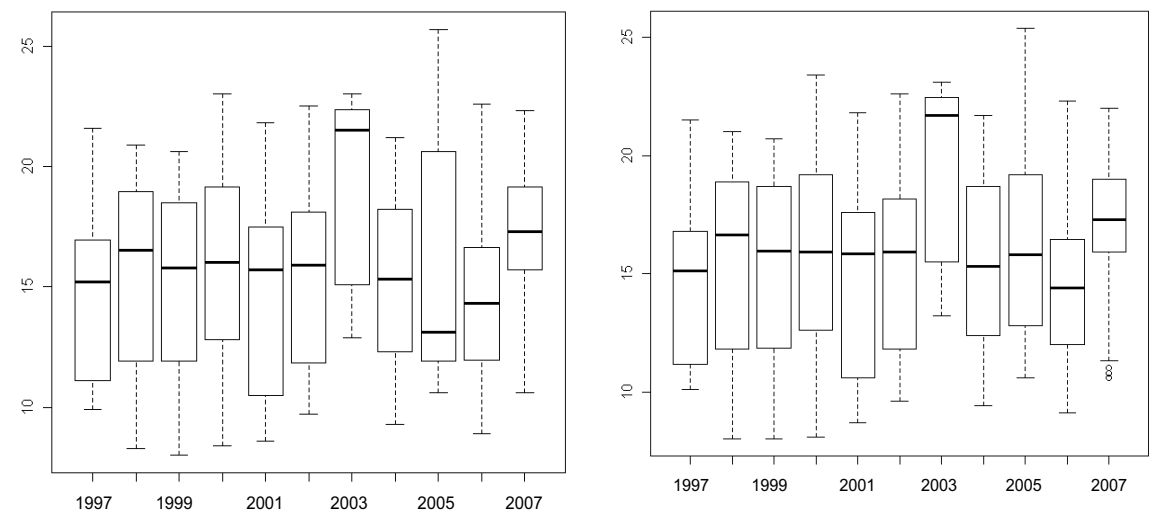

Fig. 6. Évolution de la température journalière de la Meuse (en $\left.{ }^{\circ} \mathrm{C}\right)$ d'avril à juin de 1997 à 2007 à la station SM1 amont (gauche) et aval SM3 (droite).

Fig. 6. Annual evolution of mean daily water temperature $\left({ }^{\circ} \mathrm{C}\right)$ in April-June period from 1997 to 2007 at upstream SM1 (left) and downstream SM3 (right) stations.

amont avec 271 valeurs au lieu de 365 (données manquantes en mai, juin et juillet).

Seule la température médiane à l'aval est en augmentation significative sur la période 1997-2007 d'après les tests de tendance ( $p$-value 0,0023 ).

Pour la période printanière (avril-juin), seul le quantile $25 \%$ de la température de l'eau à l'aval est en augmentation significative ( $p$-value 0.0292) sur la période 1997-2007 (Fig. 6).

En ce qui concerne la période estivale (juillet-octobre), les minima ainsi que le quantile à $75 \%$ de la température à l'aval sont en augmentation significative ( $p$-value 0,0058 et 0,0350 ) sur la chronique 1997-2007 (Fig. 7). 

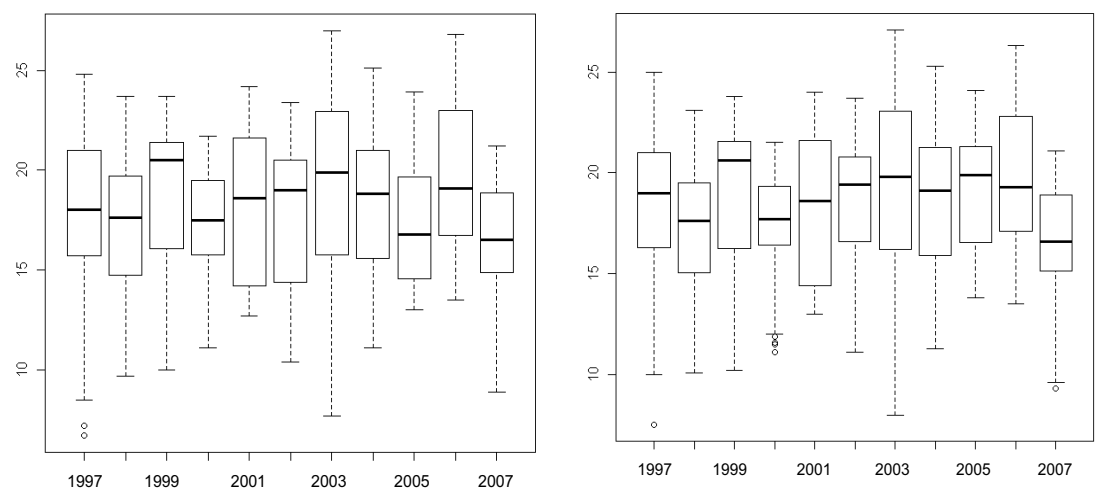

Fig. 7. Évolution de la température journalière de la Meuse $\left(\mathrm{en}^{\circ} \mathrm{C}\right)$ de 1997 à 2007 de juillet à octobre à la station amont SM1 (gauche) et aval SM3 (droite).

Fig. 7. Annual evolution of mean daily water temperature $\left({ }^{\circ} \mathrm{C}\right)$ in July-October period from 1997 to 2007 at upstream SM1 (left) and downstream SM3 (right) stations.

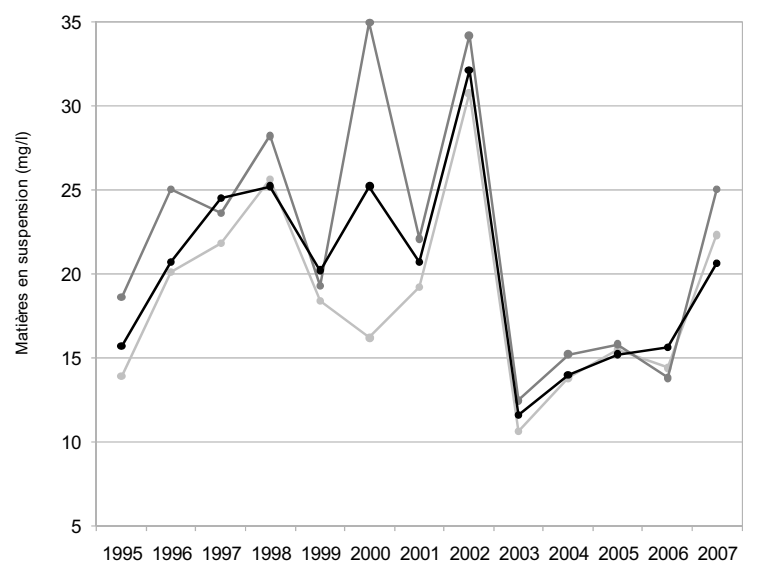

Fig. 8. Évolution de la concentration moyenne annuelle en matières en suspension (en mg/l) de 1995 à 2007 à l'amont (gris clair), l'aval immédiat (gris foncé) et l'aval éloigné (noir).

Fig. 8. Annual evolution of suspended matter concentration (mg/l) from 1995 to 2007 at upstream (grey line), downstream close to the discharge point (dark grey line) and further downstream (black line) stations.

\subsection{Paramètres de qualité d'eau}

La concentration annuelle moyenne en matières en suspension oscille entre 10 et $35 \mathrm{mg} / \mathrm{L}$ entre 1995 et 2007 (Fig. 8). L'année 2000 est très contrastée entre les stations et l'enchaînement 2002/2003 est plutôt brutal (à la baisse). Les tests de tendance indiquent que les concentrations minimales mensuelles de MES de la station aval proche sont en baisse significative entre 1995 et 2007 ( $p$-value 0,0381). 


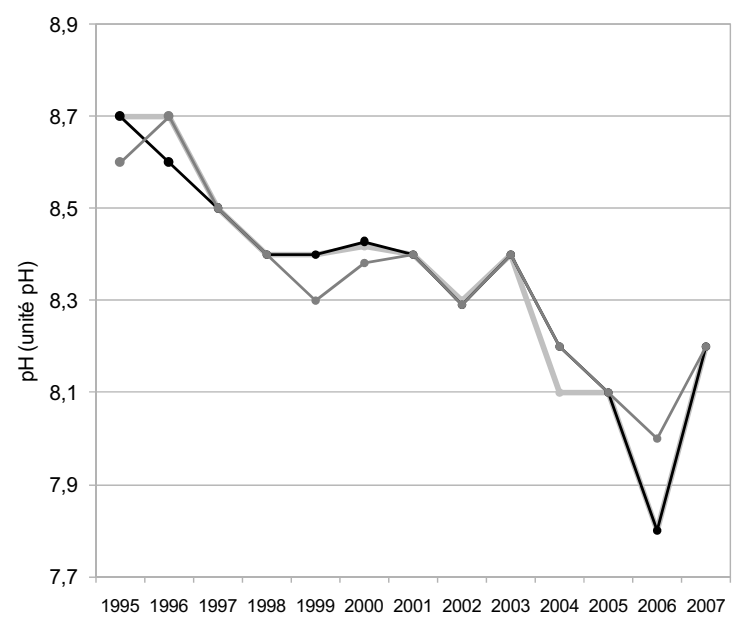

Fig. 9. Évolution du pH moyen annuel de 1995 à 2007 à l'amont (gris clair), l'aval immédiat (gris foncé) et l'aval éloigné (noir).

Fig. 9. Annual evolution of $\mathrm{pH}$ from 1995 to 2007 at upstream (grey line), downstream close to the discharge point (dark grey line) and further downstream (black line) stations.

Les valeurs de $\mathrm{pH}$ sont en baisse très significative au niveau des 3 stations de 1995 à 2007 (Fig. 9) pour toutes les données : minimum, moyenne et maximum mensuels. La Meuse est une rivière alcaline avec un $\mathrm{pH}$ oscillant entre 8,7 et 7,8 de 1995 à 2007, l'année 2006 étant la plus basse. En fin de chronique, les valeurs de $\mathrm{pH}$ caractérisent un bon état écologique de l'eau ${ }^{(1)}$.

Pratiquement tous les paramètres de DBO5 et de DCO sont en baisse significative pour les trois stations de 1995 à 2007 d'après les tests de tendance. La DBO5 varie entre 4,5 et $1,5 \mathrm{mg} / \mathrm{L}$ de 1995 à 2007, l'année 2005 étant la plus basse (Fig. 10). Cet élément de qualité classe l'eau de la

(1) Arrêté du 25 janvier 2010 relatif aux méthodes et critères d'évaluation de l'état écologique, de l'état chimique et du potentiel écologique des eaux de surface.
Meuse à Chooz en bon état écologique (DBO entre 3 et $6 \mathrm{mg} / \mathrm{L}$ ) et même très bon état les dernières années (DBO inférieure à $3 \mathrm{mg} / \mathrm{L})^{(1)}$. La DCO varie de 21 à $7 \mathrm{mg} / \mathrm{L}$ de 1995 à 2007. Sur l'ensemble de la chronique, les valeurs de $D C O$ indiquent une très bonne qualité de l'eau d'après le SEQ-Eau (la DCO n'est pas reprise dans l'arrêté du 25 janvier 2010).

Les concentrations minimales en azote total aux stations aval (SM2 et SM3) sont en baisse significative de 1997 à 2007 d'après les tests de tendance ( $p$-value $<0,05)$. L'azote total oscille entre 3,4 et 2,6 mg/L (Fig. 11).

Pratiquement toutes les concentrations en phosphore total sont en baisse significative de 1995 à 2007 aux trois stations. La concentration moyenne en phosphore total oscille entre 0,18 et $0,1 \mathrm{mg} / \mathrm{L}$ de 1995 à 2007 (Fig. 11) caractérisant un bon état écologique de l'eau ${ }^{(1)}$. 

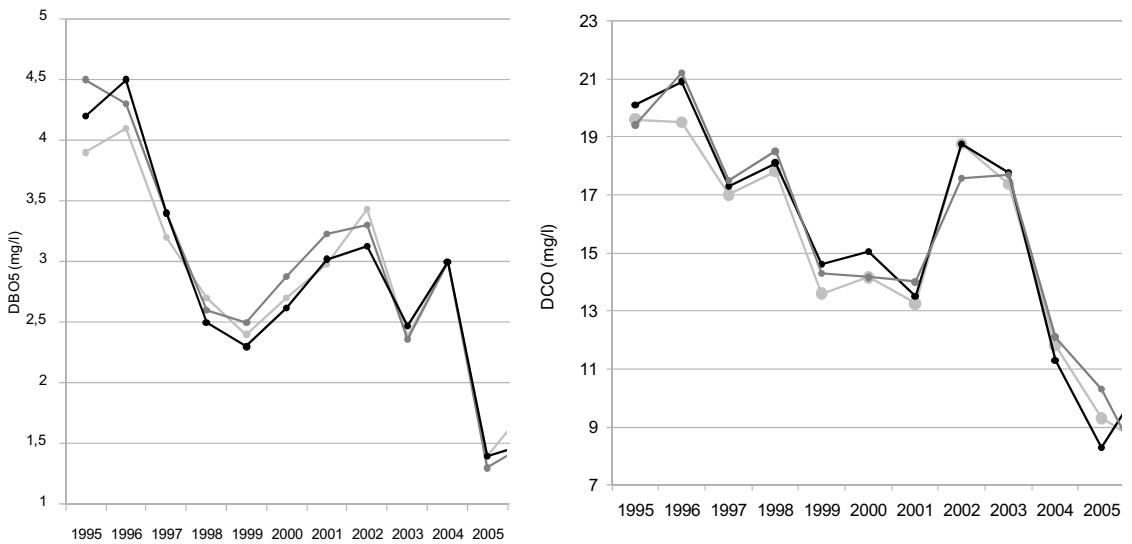

Fig. 10. Évolution de la DBO5 (à gauche) et de la DCO (à droite) en moyenne annuelle de 1995 à 2007 à l'amont (gris clair), l'aval immédiat (gris foncé) et l'aval éloigné (noir).

Fig. 10. Annual evolution of DBO5 (left) and DCO (right) from 1995 to 2007 at upstream (grey line), downstream close to the discharge point (dark grey line) and further downstream (black line) stations.
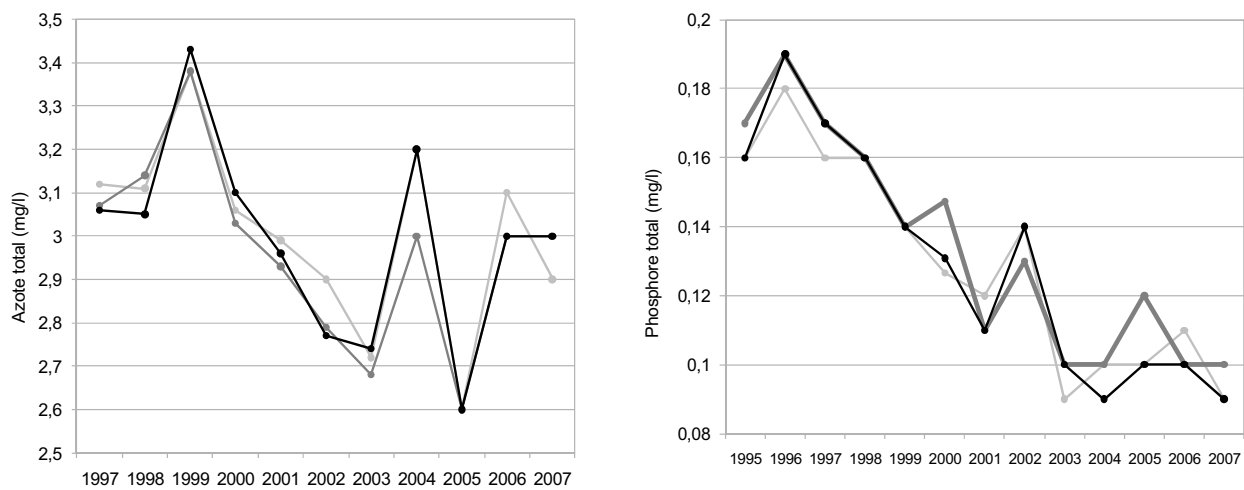

Fig. 11. Évolution de l'azote total (gauche) de 1997 à 2007 et du phosphore total de 1995 à 2007 (à droite) en moyenne annuelle à l'amont (gris clair), l'aval immédiat (gris foncé) et l'aval éloigné (noir).

Fig. 11. Annual evolution of total nitrogen (left) from 1997 to 2007 and total phosphorus (right) from 1995 to 2007 at upstream (grey line), downstream close to the discharge point (dark grey line) and further downstream (black line) stations.

\subsection{Communauté piscicole}

\subsubsection{Analyse préliminaire}

Parmi les 19 espèces piscicoles recensées entre fin août et octobre à l'amont et à l'aval de la centrale de
Chooz, 15 espèces représentant plus de $95 \%$ de l'effectif total sont considérées comme représentatives de la communauté (Tab. IV) :

- sept espèces « cœurs ", qui possèdent une forte inertie lors des tris suivant les différents critères : chevaine, 
Tableau IV. Liste des 19 espèces du peuplement piscicole de la Meuse à Chooz et des 15 retenues pour les analyses statistiques (en gris). $\mathrm{FO}=$ fréquence d'occurrence, $\mathrm{FR} 1=$ fréquence de représentativité à $1 \%$.

Table IV. List of 19 fish species of the Meuse river at Chooz and 15 species studied (grey). FO = occurence frequency, FR1 = occurence at $1 \%$.

\begin{tabular}{lllcc}
\hline \multicolumn{1}{c}{ Nom scientifique } & Nom vernaculaire & Code & $\begin{array}{c}\text { FO (\%) } \\
1991-2008\end{array}$ & $\begin{array}{c}\text { FR1 }(\%) \\
\text { 1991-2008 }\end{array}$ \\
\hline Cottus gobio & Chabot & CHA & 53 & 36 \\
Phonixus phonixus & Vairon & VAl & 31 & 19 \\
Barbatula barbatula & Loche franche & LOF & 50 & 28 \\
Leuciscus cephalus & Chevaine & CHE & 100 & 100 \\
Gobio gobio & Goujon & GOU & 97 & 92 \\
Chondrostoma nasus & Hotu & HOT & 86 & 64 \\
Barbus barbus & Barbeau fluviatile & BAF & 81 & 53 \\
Leuciscus leuciscus & Vandoise & VAN & 83 & 36 \\
Rhodeus sericeus & Bouvière & BOU & 25 & 8 \\
Rutilus rutilus & Gardon & GAR & 100 & 100 \\
Alburnus alburnus & Ablette & ABL & 94 & 86 \\
Blicca bjoerkna & Brème bordelière & BRX & 75 & 25 \\
Abramis brama & Brème commune & & & \\
Gymnocephalus cernua & Grémille & GRE & 58 & 3 \\
Gasterosteus aculeatus & Epinoche & EPI & 25 & 0 \\
Tinca tinca & Tanche & TAN & 33 & 0 \\
Scardinius erythrophatalmus & Rotengle & ROT & 14 & 3 \\
Perca fluviatilis & Perche fluviatile & PER & 94 & 69 \\
Esox lucius & Brochet & BRO & 39 & 0 \\
Anguilla anguilla & Anguille & ANG & 89 & 31 \\
\hline
\end{tabular}

gardon, goujon, ablette, hotu, barbeau fluviatile et perche fluviatile ;

- trois espèces « accompagnatrices » des espèces «cœurs»: vairon, vandoise et loche franche ;

- cinq espèces qui apparaissent et disparaissent suivant le critère ou la période considérée mais qui sont constituantes de la communauté : chabot, anguille, les brèmes, la grémille et la bouvière. Les 2 espèces de brème (bordelière et commune) ont été regroupées car leur détermination sur le terrain est difficile pour les juvéniles.

Parmi les 15 espèces retenues pour les traitements statistiques, 2 sont classées Natura 2000 (le chabot et la bouvière) et l'anguille est également remarquée du fait du règlement 

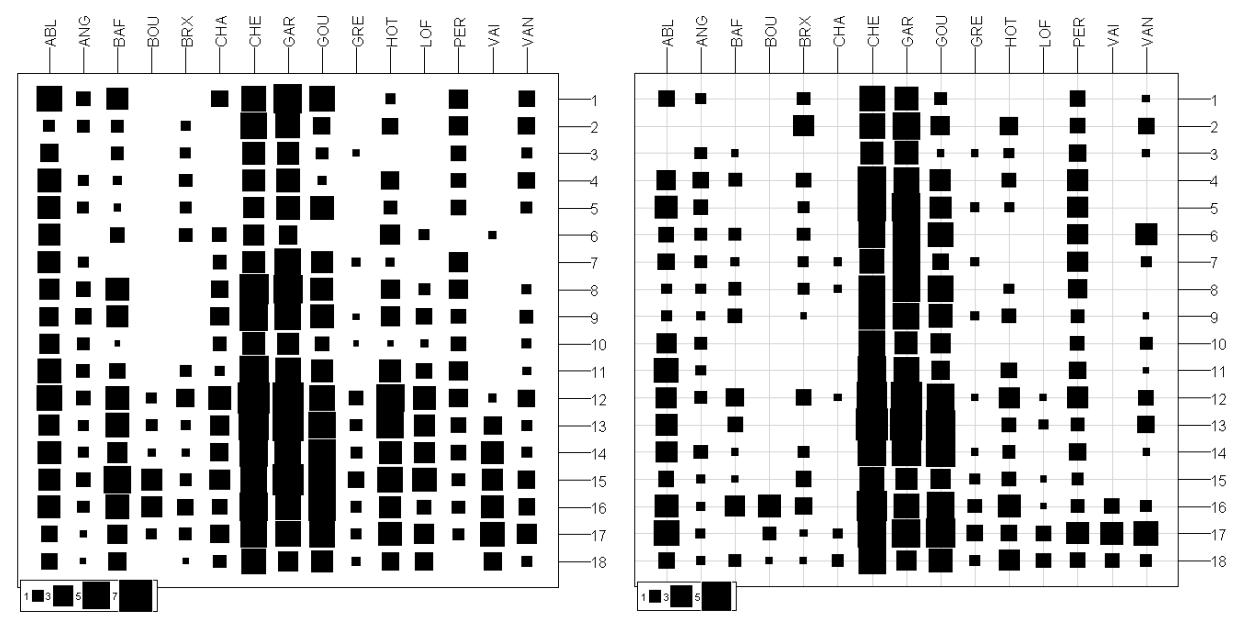

Fig. 12. Évolution des effectifs transformés $(\ln (x+1))$ de 1991 à 2008 des 15 espèces de la communauté piscicole amont (haut) et aval (bas) d'automne retenues pour l'AFC.

Abscisse: espèces suivant le code présenté dans le tableau 6. Ordonnée: années de pêche ( $1^{\text {ère }}$ année $=1991$ et année $18=2008$ ). Carrés pleins : effectif transformé d'un poisson donné à une date de pêche donnée.

Fig. 12. Evolution of $(\ln (x+1))$ abundance of 15 fish of autumnal community from 1991 to 2008 at upstream (above) and downstream (bottom) stations.

X-axis : code of the species (see Tab. 6). Y-axis : year of the sampling (1st $=1991$ and $18=2008$ ). Squares : $(\ln (x+1)) \log$ transformed abundance of fish at a given sampling date.

européen de septembre 2007 relatif à sa protection.

La figure 12 représente les abondances relatives des 15 espèces représentatives du peuplement piscicole à l'amont et à l'aval de la centrale de Chooz. Certaines espèces sont très présentes et constantes au cours de la chronique (chevaine, gardon, goujon, ablette), d'autres en revanche sont apparues plus récemment (vairon, loche franche, bouvière) et certaines ont vu leur effectif chuter (brèmes).

\subsubsection{Richesse spécifique et équitabilité}

Concernant la richesse spécifique annuelle toutes stations confondues
(Fig. 13), on constate une augmentation de l'ordre de 5 espèces passant d'environ 13 à 18 d'espèces entre 1991 et 2008. Les tests de tendance réalisés sur la richesse spécifique amont, aval et les 2 stations confondues indiquent que les évolutions sont significatives.

De 1991 jusqu'au début des années 2000 , les deux stations sont assez proches en nombre d'espèces mais ont chacune des espèces différentes l'une de l'autre. En revanche, lors des années 2000 l'écart entre les deux stations est plus grand et la station amont possède presque à elle seule l'ensemble des espèces considérées.

L'équitabilité ne présente pas de tendance significative sur l'ensemble de la chronique tant à l'amont qu'à 


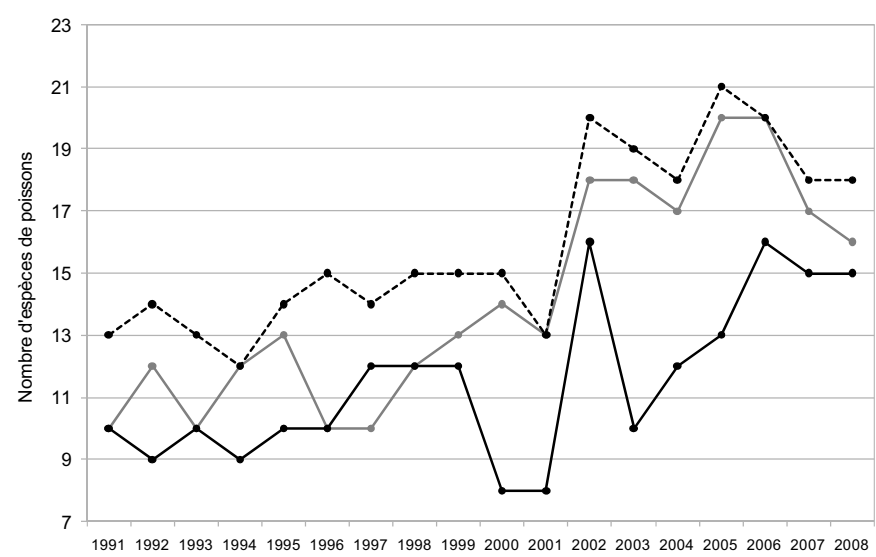

Fig. 13. Évolution de la richesse spécifique piscicole annuelle de 1991-2008 à l'amont (gris), à l'aval (noir) et toutes stations confondues (pointillés).

Fig. 13. Annual evolution of fish species richness from 1991 to 2008 at upstream (grey line), downstream (black line) and all stations (dotted line).

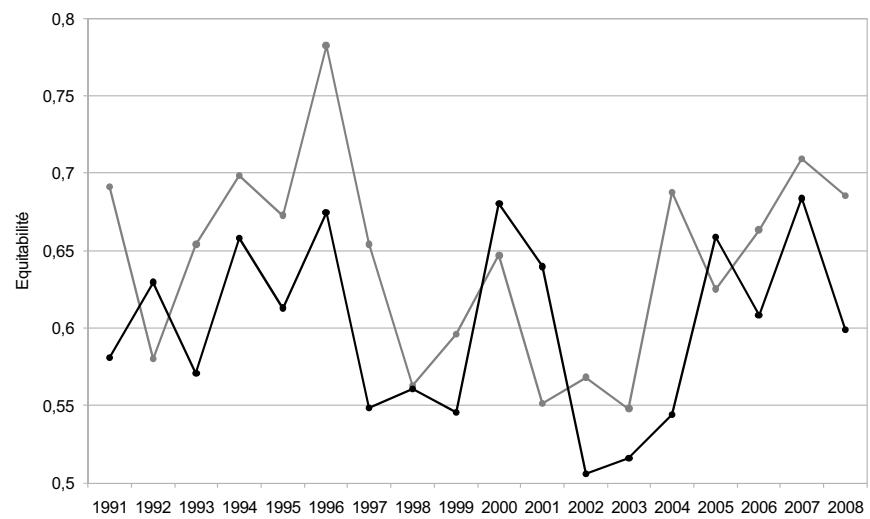

Fig. 14. Évolution de l'équitabilité annuelle sur la chronique 1991-2008 à l'amont (gris) et à l'aval (noir) de Chooz.

Fig. 14. Annual evolution of eveness from 1991 to 2008 at upstream (grey line) and downstream (black line) stations.

l'aval (Fig. 14). Elle varie entre 0,78 et 0,57 sauf pour les années autour de 1998 et 2002 qui sont très basses (entre 0,51 et 0,57 ). Une valeur inférieure à 0,60 indique un déséquilibre du peuplement avec une dominance de certaines espèces au détriment d'autres.
Nous avons vérifié si l'augmentation de la richesse spécifique à l'amont et à l'aval de la centrale de Chooz était liée à une modification du temps de pêche (Figs. 15, 16). D'après les tests statistiques, tous les paramètres (saufl'effectif total aval) augmentent significativement et aucune corrélation significative 


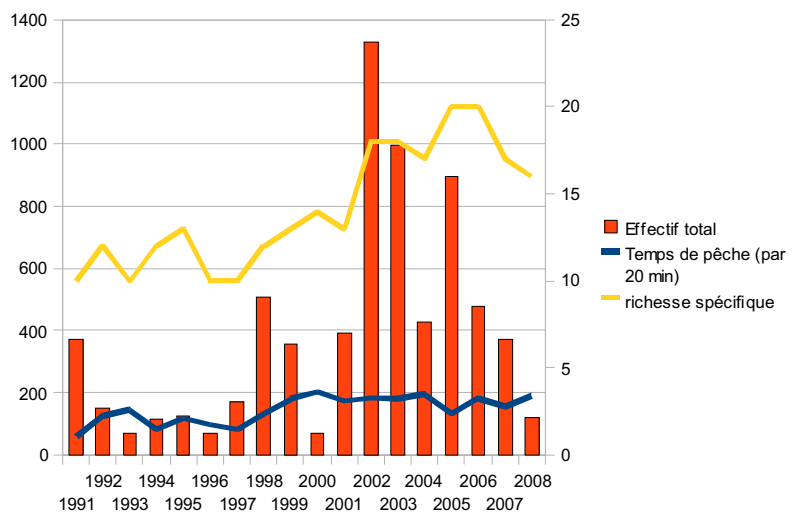

Fig. 15. Évolution de l'effectif total (nombre de poissons), du temps de pêche et de la richesse spécifique à la station amont de 1991 à 2008.

Fig. 15. Annual evolution of fish abundance, duration of fishing and species richness at the upstream station from 1991 to 2008.

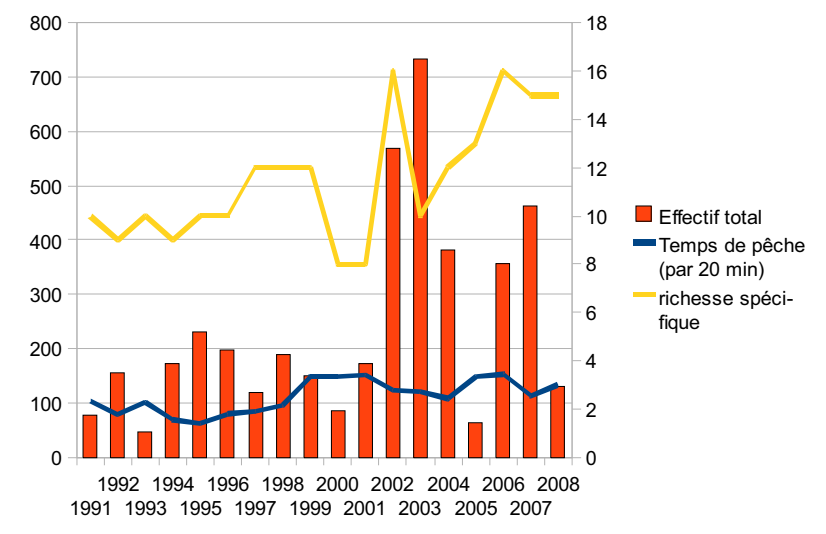

Fig. 16. Évolution de l'effectif total (nombre de poissons), du temps de pêche et de la richesse spécifique à la station aval de 1991 à 2008.

Fig. 16. Annual evolution of fish abundance, duration of fishing and species richness at the downstream station from 1991 to 2008.

n'a été trouvée, excepté entre l'effectif et la richesse spécifique à l'amont.

\subsubsection{Analyse factorielle des correspondances de la communauté piscicole}

L'analyse des deux premiers axes de l'analyse factorielle des correspondances explique $57 \%$ de la structure de la communauté piscicole automnale de 1991 à 2008. Le troisième axe apporte peu d'information complémentaire.

Le premier axe de l'AFC, qui explique près de $43 \%$ de la variabilité, est représenté sur la figure 17. La tendance d'évolution vers la partie positive de l'axe est significative pour les deux stations (amont : $p$-value $=0,0001$ et 

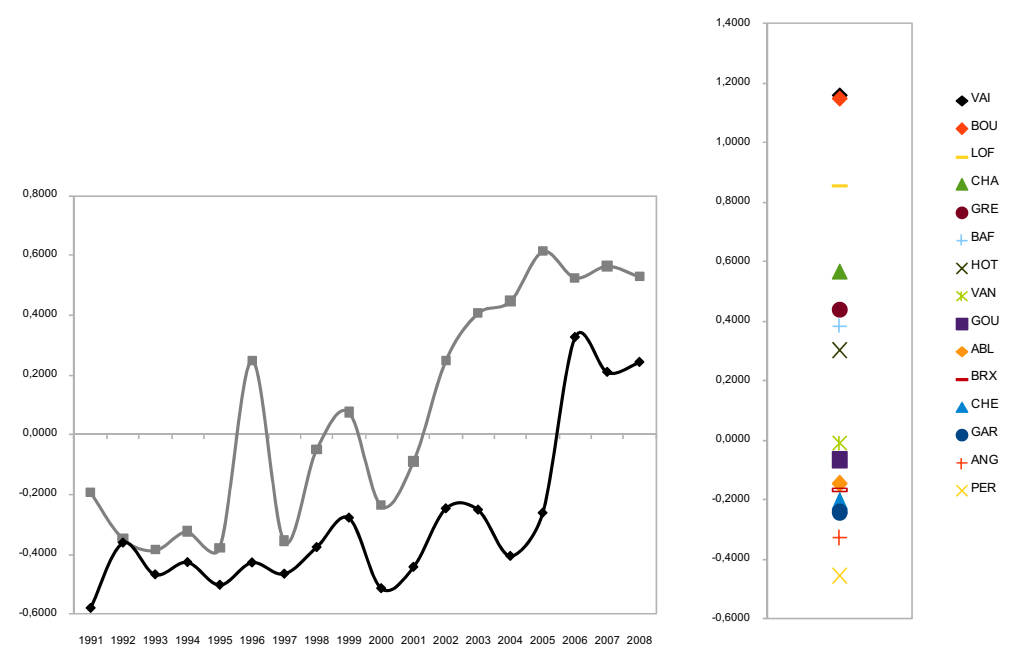

Fig. 17. Chronique des coordonnées factorielles et contribution des 15 espèces sur le premier axe de l'AFC (42,85\% de la variance) à l'amont (gris) et à l'aval (noir) de la centrale de Chooz.

Fig. 17. Annual mean scores and scores of 15 species along the first axis of COA $(42.85 \%$ of variance) at upstream (grey line) and downstream (black line) stations.

aval : $p$-value $=0,0013$ ). Le passage définitif dans la zone positive de l'axe se faisant plus tôt pour la station amont, en 2002 contre 2006 pour la station aval.

Les contributions négatives majeures pour cet axe de l'AFC proviennent de la perche, de l'anguille et dans une moindre mesure du gardon. Les contributions positives majeures pour cet axe de l'AFC proviennent, du vairon, de la bouvière, de la loche franche et dans une moindre mesure du chabot.

Le deuxième axe de l'AFC, qui explique $14 \%$ de la variabilité, ne présente pas de tendance d'évolution significative pour les deux stations.

La figure 18 qui projette les données sur les deux premiers axes de l'AFC (réunissant ainsi $57 \%$ d'explication) résume l'évolution de la structure annuelle de la communauté au cours des 18 ans de la chronique. On retrouve sur le premier axe l'opposition bouvière/ vairon/loche franche/chabot contre perche/anguille et on observe que le deuxième axe permet de dissocier la bouvière/vairon de la loche franche/ chabot.

La figure 19, qui présente les regroupements d'années structurellement similaires pour chaque station, permet d'identifier:

Pour la station amont :

- une période d'oscillation avec des années (1991-2000) où la communauté est caractérisée par le groupe de la perche et de l'anguille (ainsi que du gardon, des brèmes, de l'ablette) et des années (1996, 1998-1999 et 2001) où la communauté oscille entre les deux groupes (positif/négatif). Le deuxième axe permet de préciser que les pics positifs sont plutôt dus au chabot, à la loche franche et au barbeau fluviatile ; 


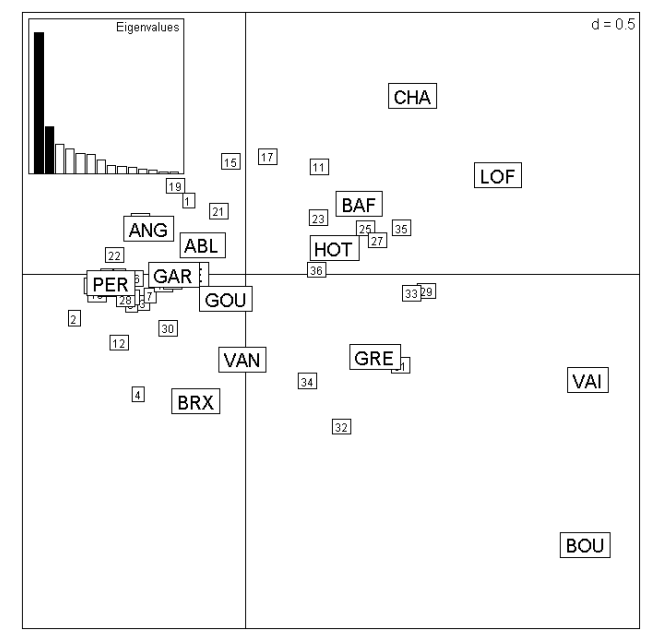

Fig. 18. Analyse factorielle des correspondances de la communauté piscicole d'automne de 1991 à 2008 ; projection sur les axes 1 (abscisse, 42,85\%) et 2 (ordonnée, 14,2\%); les codes à trois lettres correspondent aux codes poissons présentés dans le Tableau 6 ; les codes chiffrés correspondent aux numéros de pêche.

Fig. 18. COA performed on the fish community at Autumn period from 1991 to 2008 ; projection along the first $(42.85 \%)$ and the second $(14.2 \%)$ axes ; code of the species (see Table 6$)$; code of the number of sampling.

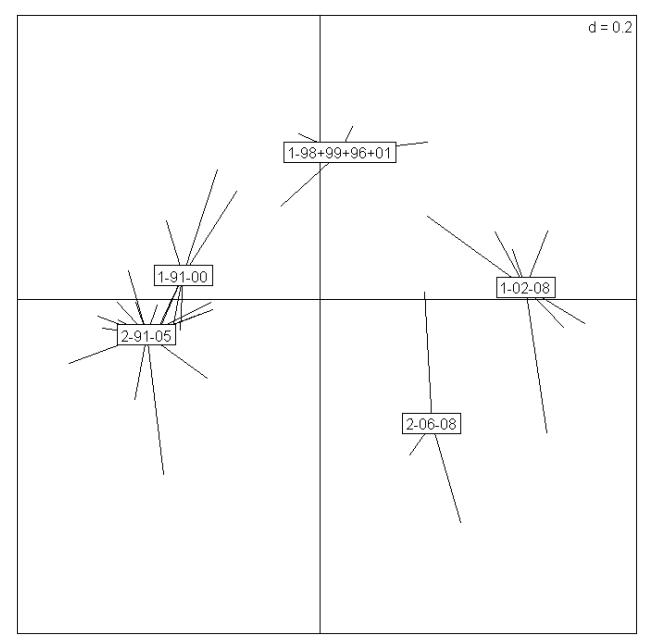

Fig. 19. Regroupement des stations amont (préfixe 1) et aval (préfixe 2) et des années structurellement similaires de l'analyse factorielle des correspondances de la communauté piscicole d'automne de 1991 à 2008 ; projection sur les axes 1 (abscisse, 42,85 \%) et 2 (ordonnée, 14,2 \%) ; les étiquettes sont les barycentres des points constituant les groupes.

Fig. 19. Groups of upstream (1) and downstream (2) stations and of the years which are similar in the COA performed on the fish autumnal community from 1991 to 2008 ; projection along the first $(42.85 \%)$ and the second (14.2\%) axes ; lines link similar samples to their stations. 
Tableau V. Résultats des tests de corrélations entre le premier axe de l'AFC de la communauté piscicole de la station amont (AFC1 amont) et aval (AFC1 aval) avec les paramètres environnementaux aux stations SM1 amont, SM2 aval proche et SM3 aval lointain ayant une tendance d'évolution significative.

Table V. Results of the correlation tests between the 1st axis COA performed on the upstream and downstream community and the physico-chemical parameters at the upstream (SM1), downstream close to the discharge point (SM2) and further downstream (SM3) stations.

\begin{tabular}{|c|c|c|c|c|}
\hline \multicolumn{2}{|c|}{ Paramètres } & Signe & $\mathrm{R}^{2}$ & $p$-value \\
\hline \multicolumn{2}{|c|}{ AFC1 amont } & & & \\
\hline $\begin{array}{c}\text { Temp. printanière aval } \\
\text { 1997-2007 }\end{array}$ & Quantile 25\% & + & 0,36 & 0,0469 \\
\hline \multicolumn{2}{|c|}{ AFC1 aval } & & & \\
\hline \multirow{2}{*}{ pH 1995-2007 } & Min SM1 & - & 0,53 & 0,0104 \\
\cline { 2 - 5 } & Min SM2 & - & 0,58 & 0,0041 \\
\cline { 2 - 5 } & Moy SM2 & - & 0,61 & 0,0270 \\
\cline { 2 - 5 } & Min SM3 & - & 0,59 & 0,0101 \\
\hline DBO5 1995-2007 & Min SM2 & - & 0,48 & 0,0200 \\
\cline { 2 - 5 } & Moy SM2 & - & 0,50 & 0,0481 \\
\hline
\end{tabular}

- une période plus nette 2002-2008: où la communauté se caractérise par le groupe de la bouvière, du vairon et de la loche franche.

Pour la station aval, 2 périodes distinctes :

- 1991-2005 : due au groupe de la perche, du gardon, des brèmes et de l'anguille ;

- 2006-2008 : due au groupe de la bouvière, du vairon et de la grémille.

\subsection{Relation entre paramètres du milieu et peuplement piscicole}

Des tests de corrélation entre les paramètres environnementaux ayant des tendances significatives ( $p$-value $\leq$ 0,05 ) et le premier axe de l'analyse factorielle des correspondances du peuplement piscicole amont et aval ont été réalisés (Tab. V).
Seule une corrélation positive entre la température printanière (quantile $25 \%$ ) de la station aval éloignée (SM3) et l'évolution du peuplement piscicole amont est mise en exergue.

En ce qui concerne l'évolution du peuplement piscicole aval, aucune corrélation n'est mise en évidence avec les paramètres de température et de débit. En revanche, le pH et la $\mathrm{DBO}_{5}$ sont inversement corrélés à l'évolution de la communauté piscicole à l'aval.

\section{DISCUSSION}

\section{1 Évolution des paramètres physico-chimiques à Chooz}

Sur la chronique 1991-2008 de la station hydrologique de Graviat, les débits ont une tendance significative à être plus rassemblés autour de la moyenne annuelle et les faibles débits 


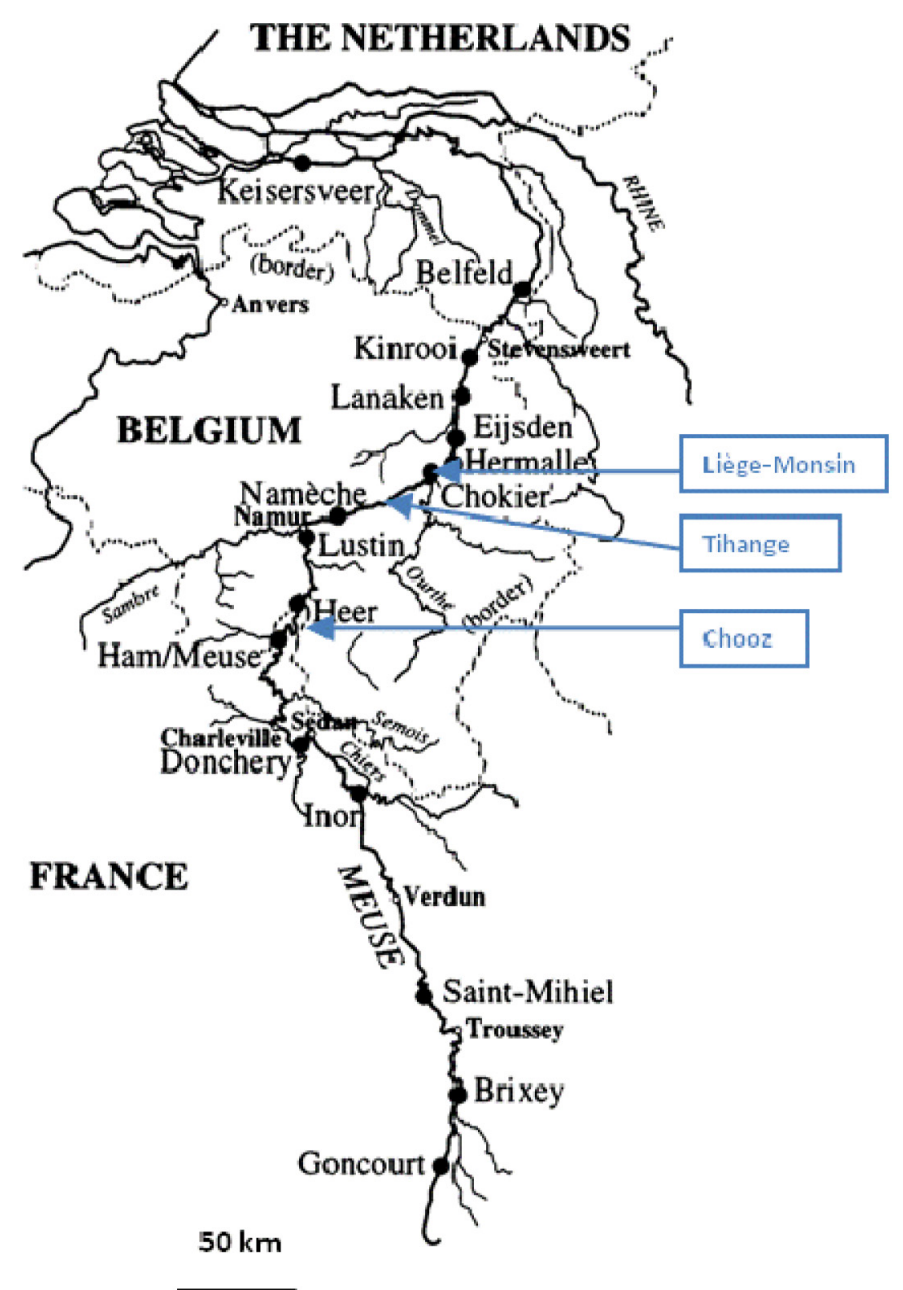

Fig. 20. Carte du bassin de la Meuse adaptée de Usseglio-Polatera \& Beisel (2002).

Fig. 20. Map of the basin of the River Meuse adapted to Usseglio-Polatera \& Beisel (2002).

sont significativement plus rares. Descy et al. (2009) ont observé une tendance à l'augmentation des débits moyens annuels de la Meuse de 1970 à 2005 d'après des mesures effectuées au niveau de trois stations (Fig. 20) : Inor (France, secteur Meuse amont), Ham-sur-Meuse (France, secteur Meuse moyenne) et Eijsden (Pays-Bas, secteur Meuse aval). Cette tendance n'est pas visible sur notre chronique plus courte.

En ce qui concerne la température moyenne de l'eau de 1997 à 2007, elle n'a pas significativement varié et oscille entre 12 et $13^{\circ} \mathrm{C}$ en amont et en aval de la centrale, l'année 2003 étant la plus chaude. L'analyse de la chronique de 
températures assez courte et incomplète apporte finalement peu d'informations. En revanche, Descy et al. (2009) disposent d'une série long-terme de température de la Meuse 1970-2005 et indiquent dans leur synthèse consacrée à la Meuse une hausse de la température moyenne annuelle de l'eau de 0,16$0,89^{\circ} \mathrm{C}$ sur les 30 dernières années à la station de Liège-Monsin (secteur aval de la Meuse, Fig. 20).

De manière globale l'ensemble des paramètres de qualité de l'eau dans la boucle de Chooz (toutes stations confondues) s'améliorent de 1995 à 2007 et caractérisent un bon état écologique. Là aussi, la chronique de données est assez courte mais des évolutions sont tout de même mises en exergue.

La baisse en matières en suspension (MES) par exemple est confirmée par Descy et al. (2009) qui disposent de suivi long-terme 1970-2005 de la concentration en MES en 2 stations sur la Meuse (Fig. 20) : Eijsden (Pays-Bas, secteur Meuse aval) et Keizersveer (Pays-Bas, environ 70 km de la mer) indiquant une diminution des MES depuis les années 1990 après une hausse durant les années 1980. Les concentrations oscillent entre 10 et $30 \mathrm{mg} / \mathrm{L}$ depuis les années 1990, ce qui est totalement cohérent avec les valeurs obtenues dans la Meuse à Chooz depuis 1995.

La diminution très nette de phosphore total entre 1995 e 2007 observée dans la Meuse via le suivi hydrobiologique de la centrale de Chooz est égalemen cohérente avec les concentrations en phosphates indiquées par Descy et al. (2009) dans la Meuse sur la période 1970-2005.
En outre, Descy et al. (2009) précisent que le niveau de pollution métallique de la Meuse a diminué au cours des dernières décennies.

Cette amélioration générale de la qualité de l'eau est également observée sur la Loire moyenne par Floury et al. (2012) suite à l'analyse des données 1977-2008 excepté pour les nitrates. Les phosphates, la chlorophylle a, la $\mathrm{DBO}_{5}$, les matières en suspension et le $\mathrm{pH}$ diminuent significativement depuis le milieu des années 80.

\section{2 Évolution de la communauté piscicole de Chooz}

\subsubsection{Structure de la communauté piscicole}

Au niveau de la boucle de Chooz, aux deux stations étudiées (amont et aval), le nombre d'espèces de poissons a significativement augmenté sur la période 1991-2008 comme dans les études menées sur le Rhône, la Loire et la Seine (Daufresne \& Boët, 2007 ; Tales, 2008).

La station Chooz amont voit son nombre d'espèces augmenter de 11 à 18 environ. Ainsi à partir des années 2000 cette station possède pratiquement l'ensemble des espèces de l'étude, elle s'est donc enrichie en espèces qui étaient auparavant uniquement présentes à la station aval.

Le faible nombre d'espèces échantillonnées à la station aval en 2000 est justifié par des conditions hydrauliques moins favorables lors de l'automne. En effet, lors de la campagne de juin 2000, le nombre d'espèces capturées à la 
station amont est le même qu'à l'aval (14 espèces). La faible richesse spécifique de l'année 2001 pour la station Chooz aval est due à des travaux à l'amont direct de la station de pêche cette année-là (remblai des berges, travaux de terrassement lourds sur les berges et en lit mineur liés à la construction d'un port). Outre ce creux, cette station est aussi plus riche en espèces en 2008 qu'au début de la chronique.

L'analyse factorielle des correspondances met en évidence une évolution significative de la communauté piscicole qui est expliquée par différentes espèces pouvant être rassemblées en trois groupes.

Le premier et principal groupe est constitué des 2 espèces qui sont apparues tout dernièrement dans la chronique (en 2002 pour l'amont et en 2006 pour l'aval) et qui représentent l'évolution majeure de la communauté (notamment pour la station amont) : le vairon et la bouvière auxquels on pourrait associer la grémille qui intervient dans les oscillations de transition de la station amont.

Le deuxième groupe est composé de deux espèces qui suivent le premier groupe en terme d'explication de l'évolution de la communauté. II s'agit d'espèces qui sont apparues assez tôt pour la station amont (1996) et plus tardivement pour la station aval : la loche franche et le chabot auxquels on pourrait associer le barbeau fluviatile et le hotu dont l'importance est moindre dans l'analyse et l'évolution de la communauté car ils sont présents tout au long de la chronique mais leurs effectifs ont augmenté, surtout à la station amont.
Le troisième et dernier groupe est représenté par les deux espèces dont l'effectif a diminué au cours de la chronique : la perche fluviatile et l'anguille auxquelles on peut ajouter les brèmes, le gardon et le chevaine.

\subsubsection{Traits biologiques des espèces}

Si l'on se réfère aux traits biologiques des espèces (Pont et al., 2011; Holzer, 2008) qui marquent l'évolution de la communauté, on distingue nettement deux périodes. La boucle de Chooz est progressivement passée d'une situation, de 1991 à 2001 pour l'amont et de 1991 à 2005 pour l'aval, où la communauté piscicole était dominée et constituée d'un «cœur " fort d'espèces de cours d'eau calme plutôt tolérantes aux variations environnementales (omnivores, eurythermes) à une situation où ce sont des espèces de cours d'eau plus vives et plus exigeantes (rhéophiles, intolérants aux basses concentrations en oxygène dissous, lithophiles) qui apparaissent. Cette évolution est très marquée et plus précoce pour le peuplement amont (dès 2002), bien moins pour l'aval qui conserve ses espèces tolérantes, en effectifs abondants, et voit apparaître plus récemment (2006) des espèces typiques des zones supérieures des cours d'eau, présentes à l'amont.

Malgré les limites du jeu de données printanier, l'analyse de la communauté printanière conforte le changement de structure de la communauté et l'implication dans ces modifications de la bouvière, du vairon, de la loche franche, du chabot et des brèmes. 
Usseglio-Polatera \& Beisel (2002) ont étudié l'assemblage des macroinvertébrés sur 16 stations réparties sur $800 \mathrm{~km}$ le long de la Meuse (Fig. 19) à partir d'inventaires réalisés en août 1998. Au niveau de la station de Ham-sur-Meuse, située à proximité de la centrale de Chooz, les assemblages observés comportent des espèces rhéophiles et d'eaux plutôt froides vivant dans des eaux claires et saturées en oxygène avec des valeurs de $\mathrm{pH}$ relativement élevées $(8,1-8,3)$. Les résultats que nous observons sur les traits des poissons sont donc cohérents avec ceux des macroinvertébrés même si dans ce dernier compartiment, cela est visible dès la fin des années 90 .

\subsubsection{Cas de la bouvière}

L'augmentation des effectifs de bouvière, espèce classée Natura 2000, dans le changement de structure de peuplement piscicole de la Meuse à Chooz a également été observée par Daufresne (2008) sur les sites de Nogent-sur-Seine, Belleville et Chinon sur la Loire, St Alban et Tricastin sur le Rhône.

Cette augmentation d'effectifs de bouvière n'est à ce stade pas bien expliquée car les bivalves Unio et Anodontes nécessaires à la reproduction particulière de cette espèce sont très peu présents dans les prélèvements de macroinvertébrés réalisés quatre fois par an lors du suivi hydrobiologique de la centrale de Chooz. En effet, trois individus d'Unio ont été recensés à l'été 1995 (station aval proche), un en mars 1996 (station aval lointain) et un en avril 2000 à la station aval. II faut tout de même noter que la technique d'échantillonnage (substrats artificiels) reste limitée pour la récolte de certains invertébrés benthiques et que la profondeur du cours d'eau empêche également leur récolte et observation.

Philippe et al. (2009) ont réalisé un inventaire de la faune et de la flore aquatiques et terrestres de la Meuse dans le cadre de la reconstruction de barrages gérés par VNF dont celui de Ham-sur-Meuse situé juste en amont de la centrale de Chooz. Dans le rapport de synthèse, il est précisé que la Mulette épaisse (Unio crassus) est observée sur la Meuse en aval de l'aire d'étude c'est-à-dire en aval de Givet, et pourrait être présente plus en amont.

Face à la hausse parallèle des effectifs de bouvières et de Corbicula dans différents bassins, la question de savoir s'il était possible que la bouvière puisse utiliser la corbicule s'est posée (Carrel, comm. pers., 2012). Corbicula fluminea a été détectée dans la Meuse à l'aval de Chooz au début des années 2000 (Brancotte \& Vincent, 2002) et est régulièrement présente de 2003 à 2007 dans les prélèvements de macroinvertébrés réalisés lors du suivi hydrobiologique de la centrale de Chooz. Mais d'après les travaux expérimentaux réalisés en Chine par Reichard et al. (2007), aucune des espèces de bouvières étudiées n'a utilisé la corbicule.

\subsection{Protocole d'échantillonnage}

Même s'il a été vérifié statistiquement que l'augmentation du temps de pêche au cours du temps n'était pas 
corrélée avec l'effectif total pêché et la richesse spécifique, le changement de protocole de pêche a peut être eu une influence sur la structure de la communauté piscicole étudiée à partir des inventaires. En effet, la pêche en continu sur de grands linéaires réalisée au début de la chronique ne permettait pas de prendre tous les juvéniles, l'effort de capture était orienté vers les gros individus. Avec la pêche par ambiance de 1998 à 2006, cela avait tendance à s'estomper puisque les longueurs pêchées étaient réduites et une part plus importante de juvéniles était capturée. La pêche par points effectuée depuis 2007 permet de lever ce biais. Le changement d'opérateurs (quatre équipes se sont succédées) peut également biaiser les résultats.

\subsection{Relation entre les paramètres du milieu et la communauté piscicole à Chooz}

Ce travail a consisté à chercher des corrélations uniquement entre les paramètres ayant des tendances d'évolution statistiquement significatives ( $p$-value $\leq$ $0,05)$ sur l'ensemble des chroniques étudiées. Excepté pour les valeurs de débit, le jeu de données environnementales est finalement assez limité (à partir de 1995 ou 1997) et il s'avère peu évident d'en tirer des conclusions.

Concernant le débit et la température, pratiquement aucun des paramètres testés n'est corrélé à l'évolution de la communauté piscicole. En effet, seule l'augmentation du quantile $25 \%$ de la température printanière de la station aval éloignée est corrélée à l'évolution du peuplement piscicole amont.
La chronique des données de température à l'amont n'étant pas complète, cela explique sans doute que ce soit celles de l'aval qui soient corrélées avec le peuplement de poissons amont.

Certains épisodes ponctuels peuvent être mis en perspective de l'évolution de la communauté piscicole. Ainsi, 2001 qui correspond au changement de structure du peuplement amont suit une année sans extremum où le débit journalier moyen est élevé (supérieur au module inter-annuel). II semble de manière générale que les espèces plutôt caractéristiques des zones supérieures des cours d'eau soient présentes lorsque les paramètres de débits sont plutôt modérés.

En ce qui concerne l'évolution du peuplement piscicole aval, aucune corrélation n'est mise en évidence avec les paramètres de température et de débit testés. Les paramètres de la qualité d'eau en revanche, et plus particulièrement la baisse de la $\mathrm{DBO}_{5}$ et du $\mathrm{pH}$, sont significativement corrélés à l'évolution de la communauté piscicole aval de la centrale de Chooz malgré la chronique assez courte de données.

Ces résultats sont en accord avec Durance \& Ormerod (2009) qui mettent en avant les effets prépondérants de l'amélioration de la qualité de l'eau et les modifications de débit par rapport à l'augmentation de la température sur la structure des communautés de macroinvertébrés de 50 cours d'eau anglais.

\section{CONCLUSION}

La richesse spécifique et la structure du peuplement piscicole de la Meuse évoluent significativement sur 
la période 1991-2008 aussi bien à l'amont qu'à l'aval de la centrale nucléaire de Chooz.

Les tendances d'évolution observées sur les poissons ne sont pas expliquées par les paramètres de débit et de température de l'eau testés (journalier moyen et extrême) mais plutôt par l'amélioration de la qualité physicochimique de la Meuse. Comme le suggèrent Durance \& Ormerod (2009), lors des analyses long-terme de peuplements aquatiques, il faut désormais considérer l'ensemble des changements globaux et pas uniquement le réchauffement climatique pour mieux expliquer leurs tendances d'évolution.

En terme de perspective, il serait intéressant de vérifier si les changements observés dans la communauté piscicole sont également visibles dans d'autres compartiments biologiques suivis à Chooz tels que les macroinvertébrés benthiques. II serait également instructif de voir si de tels suivis hydrobiologiques existent en d'autres points de la Meuse et notamment à la centrale nucléaire de Tihange plus en aval sur la Meuse (Belgique, Fig. 20).

Enfin, un travail plus poussé sur des épisodes particuliers de température et de débit (nombre de jours dépassant un seuil par exemple) permettrait également de compléter ces premières analyses.

\section{REMERCIEMENTS}

Cette synthèse a été menée dans le cadre du programme de recherche «Thermie-Hydrobiologie » EDF-IRSTEA (2008-2012). Les auteurs remercient Yves Souchon et Bertrand Villeneuve
(IRSTEA Lyon) pour leurs conseils dans le déroulement de l'étude et l'analyse des données, Mathieu Floury (EDF R\&D) pour son aide dans l'interprétation des données, Olivier Delaigue (IRSTEA Antony) pour ses informations relatives aux traits biologiques des espèces piscicoles et Georges Carrel (IRSTEA Aix-enProvence) pour ses éléments de réponse sur les relations bouvière-corbicule.

\section{BIBLIOGRAPHIE}

Brancotte V. \& Vincent T., 2002. L'invasion du réseau hydrographique français par les mollusques Corbicula spp. Modalité de colonisation et rôle prépondérant des canaux de navigation. Bull. Fr. Pêche Piscic. 365/366 : 325-337.

Cattanéo F., Carrel G., Lamouroux N. \& Breil P., 2001. Relationship between hydrology and cyprinid reproductive success in the lower Rhône at Montélimar. Archiv Für Hydrobiologie 151 : 427-450.

Chessel D., Dufour A.B. \& Thioulouse J., 2004. The ade4 package - I : One-table methods. $R$ news 4.

Daufresne M., 2004. Approche multiéchelles des relations dynamiques entre les organismes aquatiques et leur environnement, Thèse de doctorat Cemagref, Université de Lyon 1, 175 p.

Daufresne M., 2008. Impacts des pressions climatiques et non climatiques sur les communautés piscicoles de grands fleuves français. Hydroécol. Appli. 16 : 109-134.

Daufresne M. \& Boët P., 2007. Climate change impacts on structure and diversity of fish communities in rivers. Global Change Biology 13 : 1-12.

Daufresne M., Roger M.C., Capra H. \& Lamouroux N., 2004. Long-term changes within the invertebrate and fish 
communities of the Upper Rhône River: effects of climatic factors. Global Change Biology 10 : 124-140.

Delattre C. \& Souchon Y., 2008. Stratégie de recherche et programme d'actions en thermie et hydrobiologie, Rapport EDF, H-P76-2008-01604, $32 \mathrm{p}$.

Delpech C., 2007. Évolution à long terme de la structure des communautés piscicoles estuariennes. Effets de la variabilité hydroclimatique. Rapport de stage M2 Systèmes en Écologie, Université de Bordeaux 1, $25 \mathrm{p}$.

Descy JP., Kestemont P., Everbecq E., Verniers G., Usseglio-Polatera P., Gérard P., Viroux L., Beisel J-N. \& Smitz J., 2009. The Meuse River Basin (Chap. 5.1). In: Tockner K., Robinson C.T., Uehlinger U. (eds). Rivers of Europe, Academic Press, pp. 154-166.

Dessaix J. \& Fruget JF., 2008. Évolution des peuplements de crustacés du Rhône Moyen au cours des 20 dernières années, relation avec la variabilité hydroclimatique. Hydroécol. Appl. 16 : 1-27.

Dubost Environnement et Milieux Aquatiques, 2009. Suivi hydrobiologique de la Meuse au droit du CNPE de Chooz B en 2008. Rapport, 140 p.

Durance I. \& Ormerod S., 2009. Trends in water quality and discharge confound longterm warming effects on river macroinvertebrates. Freshwater Biology 54, 388405.

Floury M., Delattre C., Ormerod S.J. Souchon Y., 2012. Global versus local change effects on a large European river. Science of the Total Environment 441, 220-229.

Grenouillet G., Hugueny B., Carrel G., Olivier J.M. \& Pont D., 2001. Large-scale synchrony and inter-annual variability in roach recruitment in the Rhône River: the relative role of climatic factors and density-dependent process. Freshwater Biology 46 : 11-26.

Hamed K.H. \& Rao A.R., 1998. A modified Mann-Kendall trend test for autocorrelated data. J. Hydrol 204 : 182-196.

Holzer S., 2008. European fish species : taxa and guilds classification regarding fish-based assessment methods. PhD thesis, Wien : Universität für Bodenkultur, $195 \mathrm{p}$.

Hughes L., 2000. Biological consequences of global warming : is the signal already apparent? Trends in Ecology and Evolution, 56-61.

IPCC, 2001. Bilan 2001 des changements climatiques : Les éléments scientifiques. Cambridge University Press, Cambridge, $91 \mathrm{p}$.

IPCC, 2007. Pachauri R.K. \& Reisinger A. (eds). Climate Change 2007: Synthesis Report. Geneva, 104 p.

IPCC, 2008. Bates B.C., Kundzewicz Z.W., Wu S. \& Palutikof J.P. (eds). Climate Change and Water. Technical Paper of the Intergovernmental Panel on Climate Change, IPCC Secretariat, Geneva, $210 \mathrm{p}$.

Khalanski M., Carrel G., Desaint B., Fruget JF., Olivier JM., Poirel A. \& Souchon Y., 2008. Étude thermique globale du Rhône - Impacts hydrobiologiques des échauffements cumulés. Hydroécol. Appl. 16 : 53-108.

Ministère de l'Écologie, de l'Énergie, du Développement Durable et de la Mer, 2009. Arrêté du 30 novembre 2009 portant homologation de la décision No. 2009-DC-0165 de l'Autorité de sûreté nucléaire du 17 novembre 2009 fixant les limites de rejets dans l'environnement des effluents liquides et gazeux des installations nucléaires de base No. 139, Nos. 144 et 163 exploitées par Electricité de France (EDF-SA) sur la commune de Chooz (département des 
Ardennes). Journal Officiel, 9 décembre 2009, $9 \mathrm{p}$.

Parmesan C. \& Yohe G., 2003. A globally coherent fingerprint of climate change impacts accross natural systems. Nature 421 : 37-42.

Philippe L., Baraille L. et Fievet E., 2009. Étude de la faune et de la flore de l'Aisne et de la Meuse dans le cadre de la reconstruction de 29 barrages gérés par Vois Navigables de France. Mise à jour de l'inventaire à l'échelle de l'itinéraire Meuse. Rapport Biotope-Asconit, août 2009, $167 \mathrm{p}$.

Pihan J.-C., 2003. Suivi hydrobiologique de la Meuse au droit du CNPE de Chooz B. Université de Metz.

Pont D., Delaigue O. \& Belliard J., 2011. Programme IPR+. Révision de l'indice poisson rivière pour l'application de la DCE. Version V0 de l'indicateur. Rapport Cemagref, mai 2011, 118 p.

Pyper B.J. \& Peterman RM., 1998. Comparison of methods to account for autocorrelation in correlation analyses of fish data. Canadian Journal of Fisheries and Aquatic Sciences 55 : 2127-2140.

Reichard M., Liu H. \& Smith C., 2007. The coevolutionary relationship between bitterling fishes and freshwater mussels: insights from interspecific comparisons.
Evolutionary Ecology Research 9 : 239259.

Seegert G., Vondruska J., Lohner T. \& Dixon D., 2008. Long term monitoring of thermal effects as part of the Ohio river ecological research program (ORERP), In: Proceedings: Second Thermal Ecology and Regulation Workshop: October 2-3 (EPRI), pp. 281-292.

Sen P.K., 1968. Estimates of the regression coefficient based on Kendall's tau. Journal of the American Statistical Association 63 : 1379-1389.

Tales E., 2008. Tendances d'évolution des peuplements de poissons de la Seine en réponse à la variabilité hydroclimatique. Hydroécol. Appl. 16 : 29-52.

Thioulouse J. \& Dray S., 2007. Interactive multivariate data analysis in $\mathrm{R}$ with the ade4 and ade4TkGUI packages. Journal of Statistical Software 5.

Usseglio-Polatera P. \& Beisel J.-N., 2002. Longitudinal changes in macroinvertebrate assemblages in the Meuse river: anthropogenic effects versus natural change. River Research and Applications 18, 197-211.

Walther G.R., Post E., Convey P., Menzel A., Parmesan C., Beebee Trevor J.C., Fromentin J.M., Hoegh-Guldberg O. \& Bairlein F., 2002. Ecological responses to recent climate change. Nature 416 : 389-395. 